\title{
Using an Active Pixel Sensor in a vertex detector
}

\author{
Howard S. Matis, ${ }^{a}$ Fred Bieser, ${ }^{a}$ Yandong Chen, ${ }^{\mathrm{b}}$ Robin Gareus, ${ }^{\mathrm{a}}$ Stuart Kleinfelder, \\ Markus Oldenburg, ${ }^{a}$ Fabrice Retiere, ${ }^{a}$ Hans Georg Ritter, ${ }^{a}$ Howard H. Wieman, ${ }^{a}$ Samuel \\ E. Wurzel, ${ }^{\mathrm{a}}$ Eugene Yamamoto ${ }^{\mathrm{a}}$ \\ ${ }^{a}$ Nuclear Science Division, Lawrence Berkeley National Laboratory, 1 Cyclotron Rd., Berkeley, CA 94720, USA \\ ${ }^{b}$ Department of Electrical Engineering and Computer Science, University of California, Irvine, CA 92697, USA
}

Elsevier use only: Received date here

\begin{abstract}
Research has shown that Active Pixel CMOS sensors can detect charged particles. We have been studying whether this process can be used in a collider environment. In particular, we studied the effect of radiation with $55 \mathrm{MeV}$ protons. These results show that a fluence of about $2 \times 10^{12}$ protons $/ \mathrm{cm}^{2}$ reduces the signal by a factor of two while the noise increases by $25 \%$. A measurement 6 months after exposure shows that the silicon lattice naturally repairs itself. Heating the silicon to $100 \mathrm{C}$ reduced the shot noise and increased the collected charge. CMOS sensors have a reduced signal to noise ratio per pixel because charge diffuses to neighboring pixels. We have constructed a photogate to see if this structure can collect more charge per pixel. Results show that a photogate does collect charge in fewer pixels, but it takes about $15 \mathrm{~ms}$ to collect all of the electrons produced by a pulse of light. (C) 2004 Elsevier Science. All rights reserved
\end{abstract}

Keywords: Vertex detector; CMOS; APS; radiation damage; photogate;

\section{Introduction}

Vertex detectors are used in a collider detector to provide tracking information to decide whether a track comes from the collision point or from a secondary decay. With an impact resolution of the order of tens of microns, they can identify charmed or bottom mesons with c $\tau$ of 100 's of microns, where $\mathrm{c}$ is the speed of light and $\tau$ is the lifetime of the particle.

CMOS Active Pixel Sensors (APS) ICs [1] offer promise as the sensor for vertex detectors $[2,3]$. These ICs, which provide micron resolution, can be made very thin. They are more radiation resistant and use less power than CCDs. As these devices use a standard CMOS process, sophisticated circuits can be put on a wafer without the use of bump-bonding or wire bonds.

This paper will investigate two issues for CMOS APS sensors. First, we will extend previous radiation studies done with protons. We will show how the radiation damage can be repaired. Secondly, we will examine a method of collecting charge from the epitaxial layer with a photogate.

\section{Active Pixel Sensor Chip}

The CMOS section for an APS chip, Fig. 1, has three layers. The top layer of the device has an $n+$ diffusion / n-well surrounded by a p-well region. Below it is a more lightly doped $\left(\mathrm{p}^{-}\right)$epitaxial (epi) silicon layer locating on top of the $\mathrm{p}^{+}$wafer silicon. 


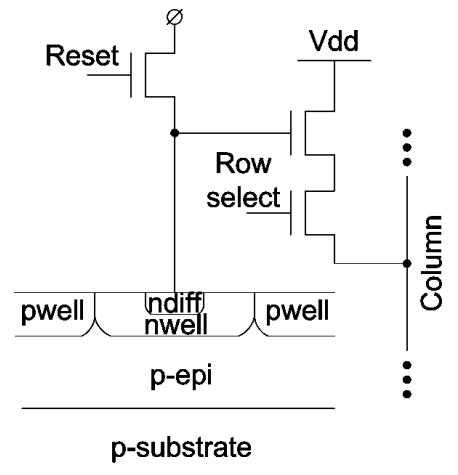

Fig. 1. This basic three-transistor active pixel sensor schematic shows a physical representation of the n-well/p-epi diode. The diagram is not to scale. The epi-layer in our design has a thickness of about $8 \mu \mathrm{m}$. The $\mathrm{N}_{\text {diff }} \mathrm{n}$-well has dimensions of an order of a micron.

When a charged particle traverses the APS IC, it creates electron-hole pairs in the epi layer. Electric fields develop at the interfaces where the doping levels change, so that electrons in the epitaxial layer are reflected at the $\mathrm{p}^{-}$well and $\mathrm{p}^{+}$interfaces. At the epi and $n$-well interface, the electric field pulls the electrons into the n-well. Since the capacitance of this diode is quite small, the voltage changes significantly for a small amount of collected charge $(\sim 30 \mu \mathrm{V} / \mathrm{e})$. The voltage, on this reverse biased floating diode formed by the $n$-well and $\mathrm{p}^{-}$epitaxial layer is read out when a column and row line address the pixel. Because the electrons diffuse in the epitaxial layer, ionization spread out over several pixels while CCDs tend to collect the charge in one or two pixels.

Two CMOS radiation sensor ICs, APS-1 and APS2, have been designed, fabricated, and tested. Results have been described earlier [4-6]. Each prototype sensor array consists of a device that is 128 by 128 pixels with a pixel size of 20 by $20 \mu \mathrm{m}$. The size of these ICs is about $2.5 \mathrm{~mm}$ per side. They were designed in a standard TSMC digital $0.25 \mu \mathrm{m}$ CMOS process that includes an 8-10 $\mu \mathrm{m}$ epitaxial layer.

\section{Radiation damage}

In a conventional $\mathrm{CCD}$, the stored electronic charge must be shifted through hundreds of pixels. Any loss of efficiency will produce a significant loss of charge. For instance, a test showed that a total of $487 \mathrm{rad}\left(3 \times 10^{9}\right.$ protons $\left./ \mathrm{cm}^{2}\right)$ with an energy range from 20 to $63 \mathrm{MeV}$ produced significant damage in a CCD [7]. In the APS design, the analog information is transferred directly from each pixel. Since the charge will only be transferred through a few pixels in the epi-layer, signal losses, which are caused by radiation traps, are minimized.

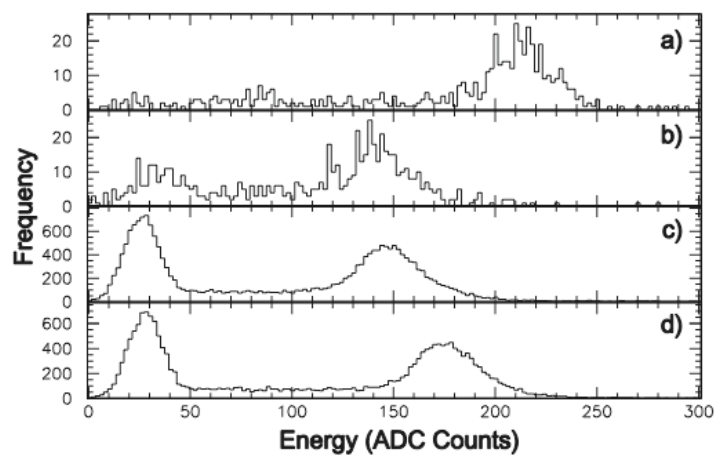

Fig. 2. Signal collected from an ${ }^{55} \mathrm{Fe}$ source. The signal is summed over nine pixels. The curves are: a) before irradiation with protons, b) after the irradiation, c) after 6 months, and d) after annealing. The peak near threshold occurs because of the noise fluctuations of the pixels. The height of the peak on the right is proportional to the source intensity. The lower the source intensity, the higher the ratio between the noise peak and the signal. As the data sets were taken various source intensities, there is a different ratio between the two peaks for each data set.

To test the effect of proton radiation, we exposed an unpowered chip to $55 \mathrm{MeV}$ protons at Lawrence Berkeley National Laboratory's 88-inch Cyclotron. Preliminary results of this test have been reported [6]. The LEPSI/IReS group has tested [8] APS chips in a neutron beam.

These results show that a fluence of about $2 \times 10^{12}$ protons $/ \mathrm{cm}^{2}$ or $300 \mathrm{krad}$ reduces the signal by a factor 
of two while the noise increases by $25 \%$. The increase in noise can be explained by the shot noise produced by the increased leakage current. Measurements show that the signal loss is produced in the epi-layer. This loss can be explained by electrons trapped by the bulk damage in the epi-layer [2].

Previous measurements on integrated circuits have shown that radiation damage can heal. We first tested an APS chip, which was exposed to $143 \mathrm{kRad}$ of radiation, 6 months after the exposure. We then annealed the chip at $100 \mathrm{C}$ for 24 hours. Fig. 2 shows a measurement of the collected signal. The radiation reduced the collected signal (summed over 9 pixels) to $65 \%$ of the original value. After 6 months, the collected charge slightly increased. After the annealing, the collected charge increased to $81 \%$ of the original charge. We did not investigate what happens if the chip were annealed for a longer time.

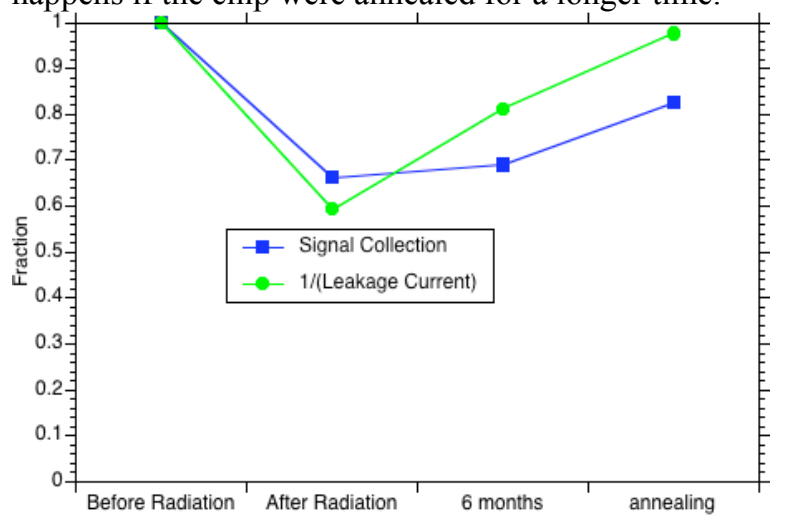

Fig. 3. Fractional change of the leakage current and the charge collected for an irradiated chip with $143 \mathrm{kRad}$. The inverse of the leakage current charge plotted.

The leakage current has a slightly different behavior. We compare the inverse of the leakage current to the signal loss in Fig. 3. This figure shows annealing by time and temperature restored the leakage current to approximately the initial value. This suggests that radiation damage produces different effects for leakage current and signal loss. We will investigate this further using $\gamma$ radiation.

\section{Photogate}

Parameters of an APS sensor, such as signal to noise and readout speed, are important when designing a vertex detector. High signal to noise is needed to suppress noise fluctuations. Correlated double sampling (CDS) is a traditional method of reducing noise, particularly $k T / C$ reset noise, but in the case of typical CMOS APS, it requires two full reads, full frame storage, and an external subtraction. The two reads slow down the readout process, so finding a method of reducing noise without this penalty is important.

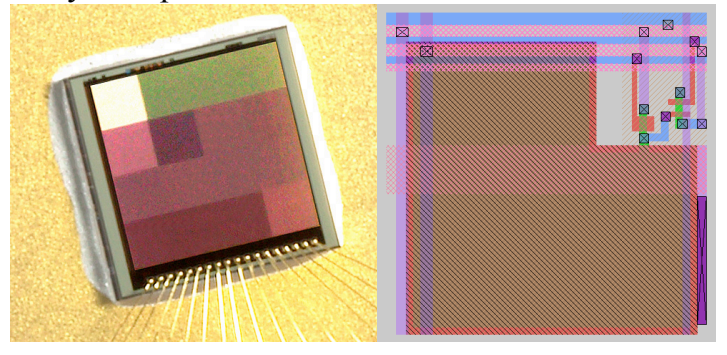

Fig. 4. Left: Photograph of AP-3, which has 16 different configurations. Sector labeling starts at the lower left corner with sector 1 and proceeds to the right. Sector 5 is just above sector 1 . Sector 1 is a photodiode circuit, while sectors, 5 and 6 are photogate circuits. Right: Sector 6 layout. The large shape is the photogate, while circuitry in the upper right corner includes the transfer gate, reset and readout transistors.

For an $8 \mu \mathrm{m}$ epitaxial layer, most probable signal amplitude with minimum ionizing particles is 450 electrons. This signal is shared among neighboring pixels. Hence, the photodiode node must have a sufficiently small capacitance to give a high charge to voltage conversion. This high gain requires that the area of the photodiode be kept small, yet this very "necessity" causes the diode to pick up an even smaller fraction of the total liberated charge in the field-free epi region. The remaining charge will diffuse out to neighboring pixels or will recombine. Thus even a best-compromise design results in a low signal to noise ratio. If charge could be collected on one pixel, an analog comparator could be used to determine when there was a hit on each pixel. A 
yes/no bit from each pixel would allow sparse readout directly on a sensor.

One way of collecting the charge in a few pixels is a photogate. This structure has been used in CCDs and light sensitive APS devices [1]. The key advantage is that the collection region of a photogate may be large, yet the collected charge is transferred to a low capacitance readout node, preserving a high charge to voltage conversion ratio.

Photogates can also make CDS more convenient, since they eliminate the requirement for complete frame storage of the first sample. Instead, two reads, one right before charge transfer, and one afterward, can take place within the readout of one row of pixels. Only one row of storage is necessary, though two reads and subtraction are still required.

A photogate collects charge in a similar manner to a photodiode. Electrons created in the epitaxial layer diffuse until they migrate to a broad field region created directly below the large area photogate, which is biased at a higher potential. They are collected and held until the photogate voltage is dropped to below the transfer gate voltage. At this point, they migrate to the higher-potential, low capacitance, readout node. The readout node must be periodically reset and the photogate must be continually cycled to keep the region directly under the photogate depleted. In principle, the photogate can deliver both higher local charge collection by virtue of its large area and high charge-to-voltage conversion gain due to the charge transfer to a low-capacitance readout node.

To study whether a photogate can be used in an APS sensor, we fabricated our third sensor - APS-3. This IC, which is pictured in Fig. 4, has $144 \times 144$ pixels with a size of $20 \mu \mathrm{m}$. The sensor, which was created in the $0.25 \mu \mathrm{m}$ TMSC process of MOSIS, is subdivided into 16 sectors. In this paper, we will discuss results from sector 1 , a circuit with one photodiode that is similar to that used in the radiation tests, and a photogate structure that can be found on sectors 5 and 6 . Some measurements on this sensor have been reported [9].

\subsection{Circuit Design}

Fig. 5 shows the photogate circuit layout. The photogate's structure differs from the standard CMOS photogate in several ways. It is a "native epitaxial silicon" photogate in that it is constructed directly in the higher-resistivity epitaxial silicon instead of lying in a p-well, as would be normal. This permits it to collect charge from the epi region. In addition, there is a small gap and no diffusion or field-oxide between the photogate and $\mathrm{N}_{\text {diff }}$ diode. The size of this gap is critical. Simulations demonstrate that too large a gap can lead to insufficient transverse field and create a potential barrier to the transfer of electrons. The distance in the fabricated circuit is $0.45 \mu \mathrm{m}$.

\subsection{Comparison with photodiode}

To compare the charge collection of a photodiode, we tested sectors 1 and 6 with an ${ }^{55} \mathrm{Fe}$ source. Fig. 6 shows the single pixel response for these two sectors. The sector 1 spectra show two components - the exponential distribution of charge from electrons deposited in the epitaxial layer and two peaks where the $\mathrm{x}$-ray deposits all its energy in the photodiode. The two peaks are produced by absorption of the ${ }^{55} \mathrm{Fe} x$ ray lines of 5.9 and $6.5 \mathrm{keV}$. Previous studies on APS IC's show that summing an array of 25 pixels produces a peak at the same value at the predominant $5.9 \mathrm{keV}$ line.

This photodiode circuit has an improved signal to noise ratio compared to the previous APS-2 circuit. By minimizing the signal traces, we were able to reduce the capacitance. We measured a noise of 10.3 electrons for a period of $1.7 \mathrm{~ms}$. This noise value includes the shot noise from the leakage current at room temperature. When we extrapolate the shot noise component from APS-2, we get a noise value of 12.7 electrons. Therefore, we have reduced the noise by $19 \%$. 


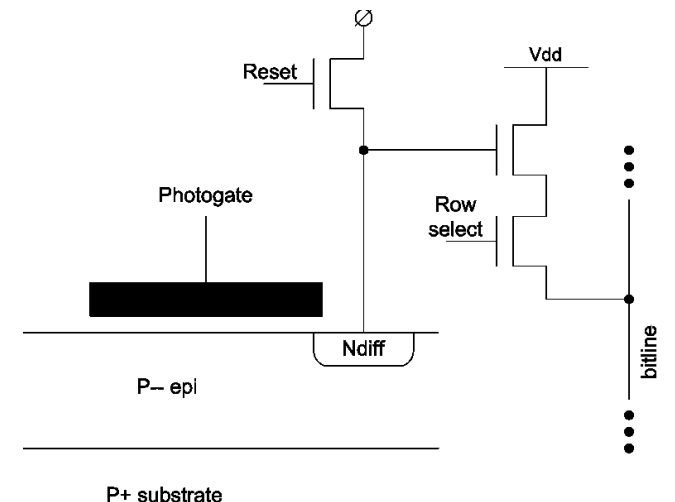

Fig. 5. Circuit diagram for the photogate. Charge that is created in the epi-layer is collected by the photogate. If the potential of the photogate is less than $\mathrm{N}_{\text {diff, }}$ then charge moves to $\mathrm{N}_{\text {diff. }}$

If a pixel in sector 6 would collect the full amount of charge, then there should be a peak in the spectrum corresponding to the full energy of the $5.9 \mathrm{keV}$ line. The photogate spectrum clearly has a larger shoulder (between 300 and 400 counts) than the photodiode circuit. Nevertheless, most of the collected charge from the photogate is measured at a lower value. Cleary, the photogate circuit is more efficient than the photodiode circuit in collecting the $5.9 \mathrm{keV}$ electrons, but there is missing charge.

\subsection{Distribution of charge among neighboring pixels}

The charge in the photogate could be contained in the neighboring pixels. To check for this, we sum the charge around the highest pixel. We find the fourpixel sum by selecting the highest value of three adjacent pixels to the central pixel. The three-pixel sum removes the lowest outside pixel and the twopixel sum uses the central pixel and the highest neighbor.

Fig. 7 shows these four sums. The four-pixel sum is essentially identical to the three-pixel sum. This shows that most of the charge is distributed among three pixels. Thus, we conclude that the $5.9 \mathrm{keV}$ line is approximately at $250 \mathrm{ADC}$ counts. Nevertheless, there are many clusters, which do not have the full energy collected. Therefore, the photogate circuit rarely collects the full charge

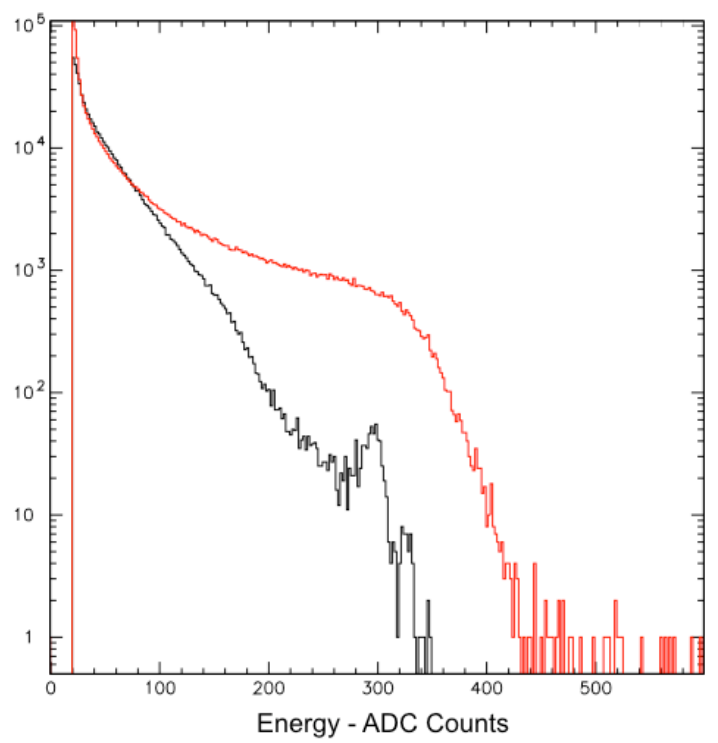

Fig. 6. The charge collected in the photogate circuit (upper curve) is compared with the charge collected by the photodiode circuit (lower curve) for a single pixel. The charge was produced by an ${ }^{55} \mathrm{Fe}$ source. The two peaks at the end of the photodiode spectra are when the $5.9 \mathrm{keV}$ and $6.5 \mathrm{keV}$ photon is absorbed by the $\mathrm{n}$ well.

\subsection{Time profile of the collected charge}

Previous measurements of an APS sensor show that charge, which diffuses in the epitaxial layer, is collected within $100 \mathrm{~ns}$ [10]. Since the readout time for an APS sensor has been in the millisecond range, the diffusion time is negligible.

To study the time structure of the photogate, we pulsed a red LED to inject charge. The results of this test can be found in Fig. 8. This graphs shows that it takes nearly $15 \mathrm{~ms}$ to accumulate the full charge. This 
long time is completely unexpected and we have no explanation.

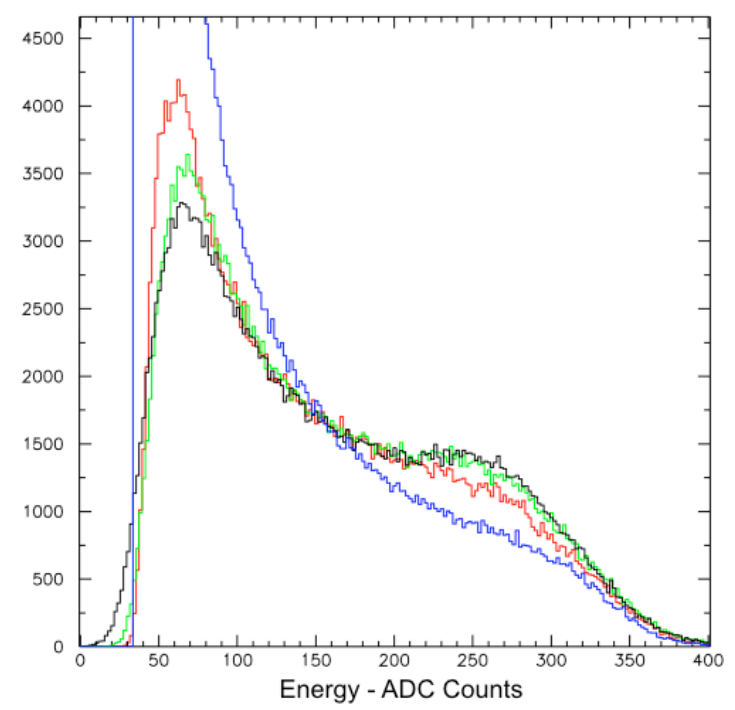

Fig.7. Distribution of charge collected for the photogate. The top curve (near 250 counts) is for summing four adjacent pixels. The curves in descending order are sums of 3,2 and 1 pixels.

However, this collection time can explain the data from Fig. 7. To obtain that data set, we read out the chip continuously. It took $1.28 \mu$ s to readout out a pixel and for this test, we read out a pixel array of 72 $x$ 72. Therefore, each pixel was read out every 6.6 ms. Since the ${ }^{55} \mathrm{Fe} x$-ray interactions occur randomly during readout, there was not enough integration time to collect the full charge.

When designing a vertex detector for an experiment, one would desire that the readout time of the detector be equal to or less than any other detector. For instance, the TPC in the STAR detector [11] has a readout time of $10 \mathrm{~ms}$ [12]. Thus, the readout time of a photogate seems too long to be used in a STAR vertex detector. We are currently planning several tests to investigate the photogates response with a scanning electron microscope.

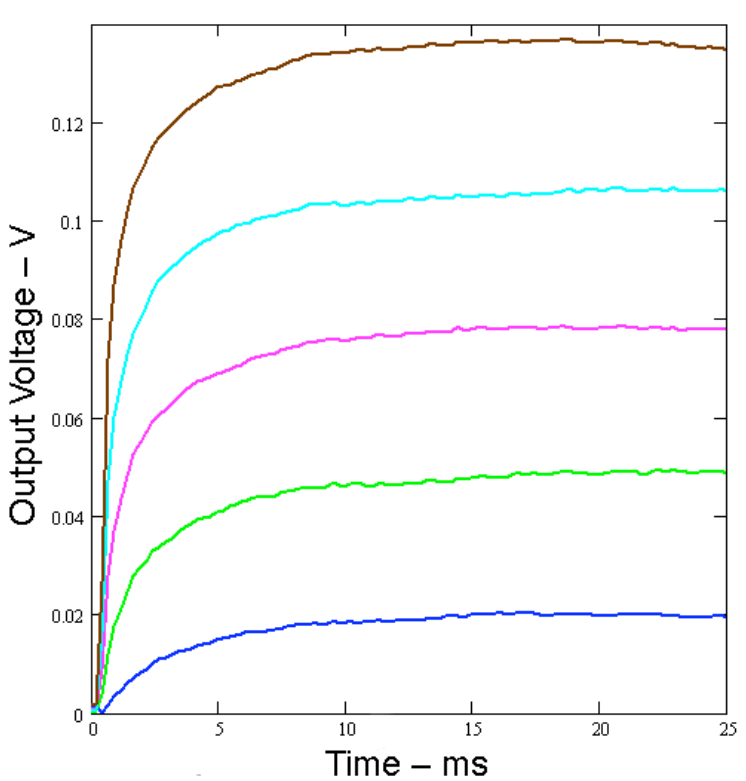

Fig. 8. Time to collect charge from a pulsed light signal for a photogate. The curves represent different amounts of charge that was injected into the pixel. The lowest curve from the bottom is the smallest value of curve. The next curve has twice the charge, while the third curve, three times the amount, etc. The leakage current is subtracted from these data sets.

\section{Summary}

As expected, APS ICs can withstand much higher doses of radiation than CCDs. Even with a $100 \mathrm{kRad}$ radiation exposure of protons, (a value much higher than a detector would receive from a RHIC experiments,) the detectors performed well. Furthermore, annealing by time and temperature reversed the radiation damage. As there is a time component in the annealing process, the damage from a continuous radiation exposure is less significant than a sudden stray beam irradiation.

Furthermore, we have designed a new APS design that has several different APS circuits. First, we have improved the noise for a photodiode circuit to 10 
electrons by improving the circuit layout. Secondly, we have explored the use of a photogate as a collector of charge. Our results show that a photogate does collect charge and the charge distribution is confined to fewer pixels than a photodiode circuit. However, it takes $15 \mathrm{~ms}$ to collect the full charge. This time is not understood and we are currently studying this effect.

\section{Acknowledgments}

The Director, Office of Science, of the U.S. Department of Energy under Contract No. DE-AC0376SF00098 supported this work in part. We would like to thank Peggy McMahan and the rest of the staff of Lawrence Berkeley National Laboratory's 88" Cyclotron for their assistance for the radiation exposure. John Wolf assembled the data acquisition board and several of our test fixtures. Kunal Singh helped with the radiation tests. We also would like to thank Hans Bichsel for his comments on this manuscript.

\section{References}

[1] E.R. Fossum, IEEE Trans. On Electron Devices 44 (1197) 168.

[2] C.J.S. Damerell, Rev. Sci. Instr. 69 (1998) 1549.

[3] G. Claus et al., Nucl. Instrum. Methods Research A, (2001) 120.

[4] H. Wieman et al., Nucl. Instr. and Meth. A 473 (2001) 205.

[5] S. Kleinfelder et al., Proc. of the SPIE, Hard X-Ray and Gamma Ray Detector Physics IV 4784 (2003) 208.

[6] H.S. Matis et. al., IEEE Trans. on Nucl. Sci. 50, (2003) 1020.

[7] C. Dale, P. Marshall and A. Burke, Proc. SPIE Vol. 1477, (1991) 70.

[8] G. Deptuch et al., Nucl. Instr. and Meth. A 511 (2003) 240.

[9] S. Kleinfelder et al., Nucl. Sci. Symposium, 2003, Conference Record of the 2003 IEEE, 2003 (in press).

[10] G. Deptuch, Ph.D. Thesis, U. Strasbourg, 2002.

[11] K.H. Ackermann et al., Nucl. Instr. and Meth. A 499 (2003) 624.

[12] M. Anderson et al., Nucl. Instr. and Meth. A 499 (2003) 679. 